\title{
Social immunity in the honey bee: do immune-challenged workers enter enforced or self-imposed exile?
}

\author{
Tarli E. Conroy ${ }^{1} \cdot$ Luke Holman $^{2}$ (i)
}

Received: 10 November 2021 / Revised: 20 January 2022 / Accepted: 24 January 2022 / Published online: 11 February 2022

(c) The Author(s) 2022

\begin{abstract}
Animals living in large colonies are especially vulnerable to infectious pathogens and may therefore have evolved additional defences. Eusocial insects supplement their physiological immune systems with 'social immunity', a set of adaptations that impedes the entrance, establishment, and spread of pathogens in the colony. We here find that honey bee workers (Apis mellifera) that had been experimentally immune-challenged with bacterial lipopolysaccharide (LPS) often exited the hive and subsequently died; some individuals were dragged out by other workers, while others appeared to leave voluntarily. In a second experiment, we found that healthy workers treated with surface chemicals from LPS-treated bees were evicted from the hive more often than controls, indicating that immune-challenged bees produce chemical cues or signals that elicit their eviction. Thirdly, we observed pairs of bees under lab conditions, and found that pairs spent more time apart when one member of the pair had received LPS, relative to controls. Our findings suggest that immune-challenged bees altruistically banish themselves, and that workers evict sick individuals which they identify using olfactory cues, putatively because of (kin) selection to limit the spread of pathogens within colonies.
\end{abstract}

\section{Significance statement}

Just as in humans, animals living in large groups must contend with infectious diseases. Social insects such as honey bees have evolved a range of behavioural and organisational defences against disease, collectively termed 'social immunity'. Here, we describe experiments in which we introduced immune-stimulated bee workers into hives to study social immunity. We find that bees that were wounded or immune-challenged were more likely to leave the hive—resulting in their death-compared to healthy controls. Some of the bees leaving the hive were ejected by other workers, while some left the hive seemingly by choice: we thus find evidence for both 'banishment' of immune-challenged bees and self-imposed exile. Furthermore, using experiments transferring chemical signals between healthy and immune stimulated bees, we establish that the latter are identified for banishment by the chemicals present on their body surface.

Keywords Cuticular hydrocarbons · Evolutionary immunology $\cdot$ Social immunity $\cdot$ Hygienic behaviour .

Lipopolysaccharide

\section{Introduction}

Colony-living animals face a heightened risk of infectious disease, which can spread rapidly when conspecifics are in

Communicated by O. Rueppell

Luke Holman

1.holman@napier.ac.uk

1 School of BioSciences, University of Melbourne, Royal Parade, Parkville, VIC 3010, Australia

2 School of Applied Sciences, Edinburgh Napier University, Edinburgh EH11 4BN, UK frequent contact (Naug and Camazine 2002; Pie et al 2004). One might therefore predict highly social species to have evolved stronger immune systems than their more solitary relatives. This prediction has received only partial support in the eusocial Hymenoptera, and indeed some evidence suggests that eusocial species have comparatively weak immune systems. Interspecific comparative analyses have found both positive and negative correlations between colony size and 
physiological immune responses (Stow et al 2007; Turnbull et al 2011; López-Uribe et al 2016), suggesting that advanced sociality does not necessarily create selection for stronger immunity, and might even select for weaker immunity.

Inspired by the lack of a clear strengthening of the immune system among eusocial taxa, as well as observations of their behaviour, researchers have proposed that eusocial insects combat pathogens using 'social immunity', which provides a complementary defence system that reduces pathogen exposure and thereby weakens selection for stronger individual immune defences (Cremer et al 2007). Social immunity is defined as the set of behavioural, physiological, and organisational adaptations that impede the entrance, establishment, and spread of pathogens in the colony (Cremer et al 2007). For example, many eusocial insects collectively remove waste from the nest (Bot et al 2001), or coat its interior with antimicrobial substances collected from plants or produced in their own bodies (Christe et al 2003; Simone-Finstrom and Spivak 2010). Many species have compartmentalised nests that help to contain the spread of pathogens; for example, leaf cutter ant nests have a separate garbage dump, and workers from the dump tend not to venture into the rest of the colony (Hart and Ratnieks 2001). Some bees deal with infestations of parasitic mites by identifying and removing infected larvae and pupae (Spivak 1996), or by 'mummifying' foreign objects or even live intruders with wax and propolis (Greco et al 2010). Asian honey bee larvae (Apis cerana) were found to die more quickly upon exposure to parasitic mites than a non-coevolved species of honey bee (A. mellifera), leading the authors to hypothesise that $A$. cerana has evolved 'social apoptosis' in order to limit within-colony parasite transmission (Page et al 2016). Pull et al. (2018) observed ant workers identifying infected ant pupae via chemical cues, and then spraying them with antimicrobial poison, in a similar manner to how diseased cells are removed by the immune system in the bodies of multicellular organisms.

Studies have also reported that honey bees show behavioural responses towards sick individuals, in ways that suggest social immunity. Baracchi et al. (2012) introduced newly emerged worker bees infected with deformed wing virus (DWV) to the hive alongside healthy controls and observed that the infected bees were frequently ejected from the hive by other workers, while controls were not. Moreover, DWV-infected bees produced a different blend of cuticular hydrocarbons, as measured by gas chromatography (Baracchi et al 2012). Cuticular hydrocarbons (CHCs) are a layer of waxy chemicals on the body surface that prevents desiccation and has many important functions in chemical communication (e.g. Richard and Hunt 2013), including in the identification of nestmates (van Zweden and d'Ettorre 2010), the queen (Holman 2018), and workers of differ ages and task specialties (Greene and Gordon 2003). Secondly, Richard et al. (2008) found that applying chemical cues extracted from the body surface of experimentally immune-challenged bees to healthy bees caused the latter to elicit more antennation and allogrooming from nestmates. Together, these results suggest that workers can detect sick nestmates (possibly via chemical cues such as $\mathrm{CHCs}$ ), and that they sometimes respond behaviourally by investigating, avoiding, and/or ejecting sick individuals.

Furthermore, kin selection theory (Hamilton 1964) predicts that some social insects might have evolved 'altruistic' responses to sickness. In advanced eusocial species like honey bees, workers rarely breed, and instead reproduce their alleles indirectly by providing assistance to relatives such as their mother queen and her offspring. Thus, as soon as the worker's presence flips from having a beneficial to a detrimental effect on the fitness of its relatives-such as when the worker picks up an infectious pathogen-leaving the colony permanently may become the course of action that maximises the worker's inclusive fitness. Consistent with this idea, Rueppell et al. (2010) observed that worker bees exposed to harmful doses of $\mathrm{CO}_{2}$ or hydroxyurea flew out of the colony and did not return; the authors hypothesised this behaviour to be 'altruistic suicide' that evolved via kin selection to prevent harm to related nestmates. Similarly, Heinze and Walter (2010) found that ants affected by a fungal infection left their nests and remained outside until death. Another study reported that experimentally immunechallenged bees showed reduced movement and reduced social interactions, and hypothesised this as an adaptation that limits disease transmission (Kazlauskas et al 2016).

In light of this research, we hypothesise that Apis mellifera honey bees (and perhaps other eusocial insects) use a multi-pronged approach to social immunity that involves both collective (e.g. eviction of sick individuals by the society) and individual behaviours (such as altruistic self-isolation). We here investigate these ideas using behavioural experiments on social immunity involving bacterial lipopolysaccharides (LPS), which are non-living cell wall components found in Gram-negative bacteria. LPS elicits a strong response from the innate immune system in many organisms, including honey bees (Imler et al 2000; Richard et al 2008; Aubert and Richard 2008; Kazlauskas et al 2016). LPS is experimentally useful because it is non-living and thus cannot manipulate host behaviour as some pathogens and parasites do (Hughes et al 2012); this is important because social immunity arises from adaptive behaviours that are attributable to hosts, not pathogens. In experiment 1 , we treated worker bees with LPS or two different procedural controls, re-introduced them to their natal hive, and then estimated the rates at which bees (1) remained inside, (2) left voluntarily, or (3) were ejected by other workers. In experiment 2 , we transferred surface chemicals from immune-challenged 
bees to healthy bees and tested whether the healthy bees were evicted from the hive more often than controls that were treated with chemicals from healthy bees. In experiment 3, we observed pairs of bees in which one member was immune-challenged, to test whether they spent more or less time in close proximity relative to control pairs in which one member was treated with sterile saline.

\section{Methods}

\section{Experimental animals}

We utilised five honey bee colonies, each housed in a Langstroth hive on the Parkville campus of The University of Melbourne. Most of the work was carried out in April-June 2019, except one block of experiment 1 conducted in December 2019. The workers used in our experiments were collected by opening up a hive and selecting a centrally located frame containing developing brood, then brushing a haphazardly selected sample of workers clinging to the frame into a plastic container for transport to the lab. By selecting within-nest bees that did not fly in response to the disturbance of opening the hive, we aimed to preferentially collect younger bees that have not yet begun performing outside-nest tasks such as guarding and foraging. Therefore, our default expectation is that most of these bees would remain inside when returned to the hive. The code supplement to this paper includes all raw data and presents tables of all sample sizes for each experiment split by treatment and hive (lukeholman.github.io/social_immunity).

\section{Experimental immune challenge and controls}

We diluted LPS (from serotype 055:B5 E. coli; SigmaAldrich) to $0.5 \mathrm{mg} / \mathrm{mL}$ in a sterile physiological saline (Ringer's solution, prepared from autoclaved, double-distilled water), and then stored it in aliquots at $-18{ }^{\circ} \mathrm{C}$ prior to the experiments (the serotype and concentration follow Richard et al 2008). For all three experiments, we used Ringer's solution with no added LPS (henceforth 'Ringers') as a control for the process of wounding bees with saline; aliquots of Ringers were prepared and frozen at the same time as the LPS-containing aliquots, from the same batch of Ringer's solution.

To administer the Ringers and LPS solutions, bees were anaesthetised in small groups (30-40 individuals) by placing them in $\mathrm{a}-18{ }^{\circ} \mathrm{C}$ freezer inside a plastic container until they were immobile but still moving their appendages (typically $c .6 \mathrm{~min}$ ). We then kept the containers of bees over ice and monitored them to maintain this state of light anaesthesia. Using a stereomicroscope and an entomological pin $(0.25 \mathrm{~mm}$; sterilised in ethanol and a candle flame between uses), we then randomly selected a bee, dipped the pin into one of the treatment solutions, and then inserted the pin through the pleural membrane between the fourth and fifth tergal segments to a distance of roughly $1 \mathrm{~mm}$ (using a different pin for each treatment solution). Bees in experiment 1's 'Intact control' group were handled similarly (i.e. anaesthetised and manipulated under the microscope), but were not punctured with a pin. After treatment, bees were marked on the thorax with a dot of coloured paint to identify their treatment group; we used a different pairing of colours and treatments for each experimental replicate to prevent confounding. For all experiments, we applied the treatments on a rotation, preventing confounding effects from, e.g. order of processing and time under anaesthesia.

\section{Experiment 1: Do immune-challenged bees leave the hive?}

Experiment 1 utilised three treatments: an intact control, and treatments in which bees were punctured with a needle coated in Ringers or LPS. After applying the treatments, bees were housed in their treatment groups in the dark at $25^{\circ} \mathrm{C}$ for $18 \pm 1 \mathrm{~h}$. We then removed any bees that had died or showed impaired mobility, and reintroduced the remainder to the hive by opening the hive and returning them to the central frame from where they had been collected. The hive entrance was then recorded for up to $2 \mathrm{~h}$ by an observer who stood by the hive (mean observation time: $97.5 \mathrm{~min}$ ). We also video recorded the hive entrance, and a second observer verified each observation (blind to treatment).

We recorded a categorical response variable with three possible outcomes: bees either stayed inside the hive for the duration of the observation period, left the hive voluntarily, or were forced out. We recorded a bee as leaving voluntarily when it walked or flew out of the hive without interacting with any other workers (in practice, only 2 bees left by flying; the other 22 walked out). We recorded the 'forced out' outcome when the focal bee was pulled out of the hive by one or more other workers using their mandibles and thrown/ dropped from the entrance of the hive. Only bees that left the landing board at the hive entrance were classified as having exited the hive. Four bees exited the hive and then re-entered $(1 \times$ intact control, $1 \times$ Ringers, $2 \times$ LPS $)$; the meaning of this behaviour is not clear, so these four observations were excluded from analysis. Experiment 1 was replicated over four hives: three in autumn (30/04, 3/05 and 9/05 in 2019) and one in spring/summer (19/11/2019; added to increase sample size).

\section{Experiment 2: The role of cuticular odours}

Experiment 2 involved two treatments. We first collected a sample of c. 200 workers from inside a hive, anaesthetised 
them, and punctured them with either Ringers or LPS in Ringers (as in experiment 1 , except that we did not apply a paint mark). We then housed these bees at $25{ }^{\circ} \mathrm{C}$ in the dark for $24 \mathrm{~h}$ in their treatment groups, to give time for changes in the cuticular odour profile to occur in response to the experimental and control manipulations. Earlier experiments have shown that $24 \mathrm{~h}$ is sufficient for LPS-treated insects to develop a substantially different chemical profile relative to Ringers-treated controls (Holman et al 2010); indeed, Richard et al. (2008) observed changes after $4 \mathrm{~h}$.

Next, we freeze-killed the surviving bees and placed an equal number from each treatment into two 20 -mL glass vials. We then added $500 \mu \mathrm{L}$ hexane (HPLC grade; Sigma ref. 34,859 ) per bee, and then gently shook the vials by hand for $60 \mathrm{~s}$ to facilitate dissolution of hexane-soluble epicuticular chemicals, then pipetted the extract into aliquots in $2-\mathrm{mL}$ glass vials.

On the same day that we prepared the chemical extracts, we collected 200 more bees from the interior frames of the same hive, which were cold-immobilised and then marked with one of two paint colours. As before, we used different treatment-colour pairings for each hive to avoid confounding. Because hexane is toxic, we applied the extracted $\mathrm{CHCs}$ to the bees indirectly by pipetting $20 \mu \mathrm{L}$ of the appropriate hexane solution onto the surface of deionised water in a 10-mL glass beaker (Smith et al 2012). After waiting a few seconds for the hexane to evaporate, we then dipped an anaesthetised, paint-marked bee through the surface of the water, and swirled it in the water's surface to allow it to pick up hydrophobic solutes (e.g. cuticular hydrocarbons) that were floating on the water's surface. The odour-coated bees were then reintroduced to their hive, and their subsequent emergence was recorded over the next 1-2 h (mean $97.5 \mathrm{~min}$ ), as in experiment 1. Experiment 2 was replicated across four hives between 26th May and 28th June 2019.

\section{Experiment 3: 'Social distancing' following immune challenge}

Like experiment 2, this experiment had two treatments: Ringers and LPS. We collected approximately 250 bees from a brood frame as before, paired them at random, and then randomly assigned each pair to one of the two treatments. One of the bees in each pair was punctured with either Ringers or LPS, while the other was left intact. Each pair was placed into a $150-\mathrm{mm}$-long $22-\mathrm{mL}$ glass test tube stoppered with cotton wool which took up about $20 \mathrm{~mm}$ of the test tube, leaving $130 \mathrm{~mm}$ for the bees to move around in. All of the test tubes were put into a ZebraTower video recording cabinet (Viewpoint, France), then recorded under infrared illumination (invisible to bees). We then analysed the videos using 'scan sampling'; specifically, we examined video stills separated by 120 -s intervals, and recorded whether or not each pair of bees was in close contact (defined as within $1.5 \mathrm{~cm}$ of each other) in each video still (i.e. the response variable was binary). The observation period lasted $3.5 \mathrm{~h}$ and began $30 \mathrm{~min}$ after closing the video recording cabinet to allow time for the bees to settle following the disturbance. Videos were transcribed blind with respect to treatment and hive. Experiment 3 was replicated across four hives in the same time period as experiment 2 .

\section{Statistical analysis}

Our main statistical analyses for experiments 1 and 2 use an uncommon model type-multinomial logistic models (hereafter MLM) - which we believe is the right choice because these experiments have a categorical response variable with three possible outcomes. However, because MLM is unfamiliar, we first present a similar analysis of the experiment 1 and 2 data using a more common type of model (a binomial generalised linear mixed model; GLMM), to demonstrate that the main conclusions are not unique to the MLM. For the GLMM, we first re-coded the response variable to have only two outcomes, namely 'Stayed inside' or 'Left the hive' (i.e. the sum of the 'Forced out' and 'Left voluntarily' outcomes), then ran a GLMM with treatment as a fixed effect and hive as a random effect. GLMMs were implemented in the lme4 package for R. Note that the GLMM requires that we discard information about which bees left by force vs voluntarily, making the MLM preferrable.

The MLM is an extension of binomial models to data with $>2$ outcomes. We implemented the MLM in a Bayesian framework via the R package brms (Bürkner 2017). As in the GLMM, we included treatment as a fixed effect and hive as a random effect. We specified a prior distribution of $N\left(\mu=0, \sigma^{2}=3\right)$ for the fixed effect estimates and $N(\mu=0$, $\sigma^{2}=2$ ) for the random effects, in order to regularise the parameter estimates (see McElreath 2020) and help model convergence.

Experiment 3 had a binary response variable and was therefore modelled using a GLMM, implemented in brms. The model included treatment, hive, and the additional random factor 'pair ID', to account for repeated measurements of each pair of bees; hive was fitted as a fixed factor to ensure the model converged.

We ran four chains for the Bayesian models of experiments 1 and 2 (5000 iterations per chain; first 2500 discarded as burn-in), and confirmed model convergence and fit via $\widehat{R}$ statistics and posterior predictive plots. The model for experiment 3 required additional iterations $(20,000$ per chain, with 10,000 burn-in) to ensure adequate effective sampling. To make inferences from each model we ran 'planned contrasts', i.e. calculating the posterior estimate of the difference in means between each pair of treatments, then finding the log odds ratio (and its 95\% CIs) as a measure of effect size. All 
data and R scripts are documented at lukeholman.github.io/ social_immunity.

\section{Results}

\section{Experiment 1: Do immune-challenged bees leave the hive?}

In the preliminary GLMM, there was a significant effect of treatment (Wald $\chi^{2}$ test; $p=0.00012$ ) on the proportion of bees leaving the hive. More bees left in the LPS treatment relative to the intact control (contrast; $p<0.0001$ ), though the increase in LPS relative to the Ringers control was marginally non-significant ( $p=0.083$ ). Non-significantly, more bees left the hive in the Ringers control compared to the intact control $(p=0.086)$.
Figure 1A uses the results of the Bayesian MLM to display the posterior estimates of the percentages of bees from each treatment group that stayed inside the hive, left voluntarily, or were forced out, while Fig. 1B shows the standardised effect size for contrasts between treatment groups for each of the three response categories. Table $\mathrm{S} 1$ shows the MLM's parameter estimates, and Table S2 shows the results of planned contrasts comparing each pair of treatments (as in Fig. 1B).

Bees treated with LPS were more than twice as likely to stay inside the hive compared to the intact control. This difference was statistically significant: the posterior probability that the true effect size is negative was over $99.99 \%$ (Table S2; this percentage can be interpreted similarly to a one-tailed $p$-value of 0.0001). Hereafter, we write PP to represent 1 minus this posterior probability, for easier comparison with the familiar $p$-value. Furthermore, bees that received LPS were nonsignificantly less likely to stay inside the hive than those that received the Ringers control $(\mathrm{PP}=0.0516)$, providing weak

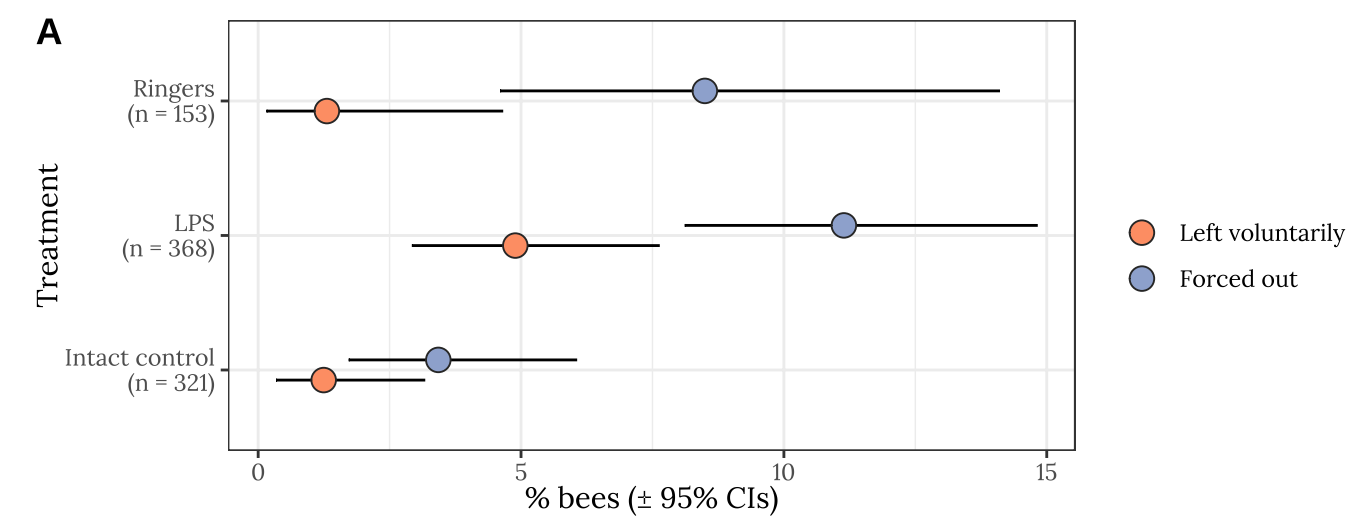

B

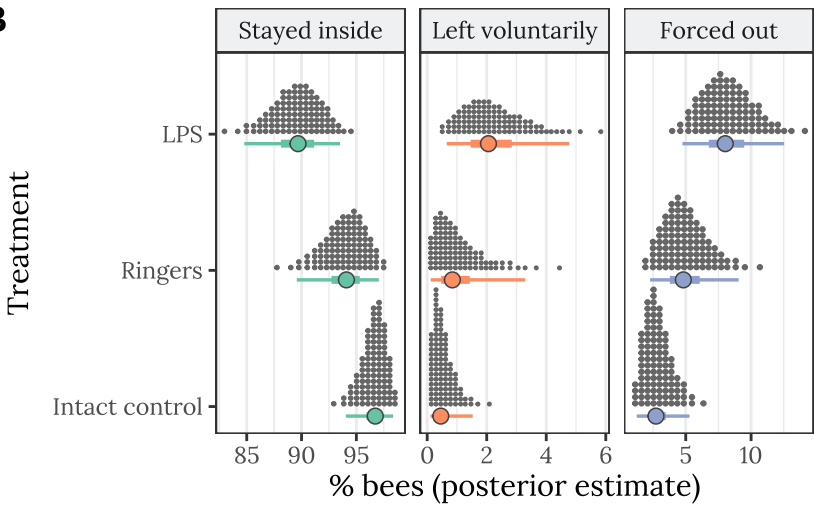

Fig. 1 Results of experiment 1 ( $n=842$ bees). Panel (A) shows summary statistics of the raw data, i.e. the percentage of bees leaving or being forced out of the hive ( $\pm 95 \%$ confidence intervals), while Panels (B) $-(\mathbf{C})$ show estimates from the Bayesian MLM. Panel (B) shows the posterior estimate of percentage of bees staying inside the hive (left), leaving voluntarily (middle), or being forced out (right), for each of the three treatments. The quantile dot plot shows 100 approximately equally likely estimates of the true percentage of bees (given the data, model, and prior), and the horizontal bars show the median and the $50 \%$ and $95 \%$ credible intervals of the posterior dis-

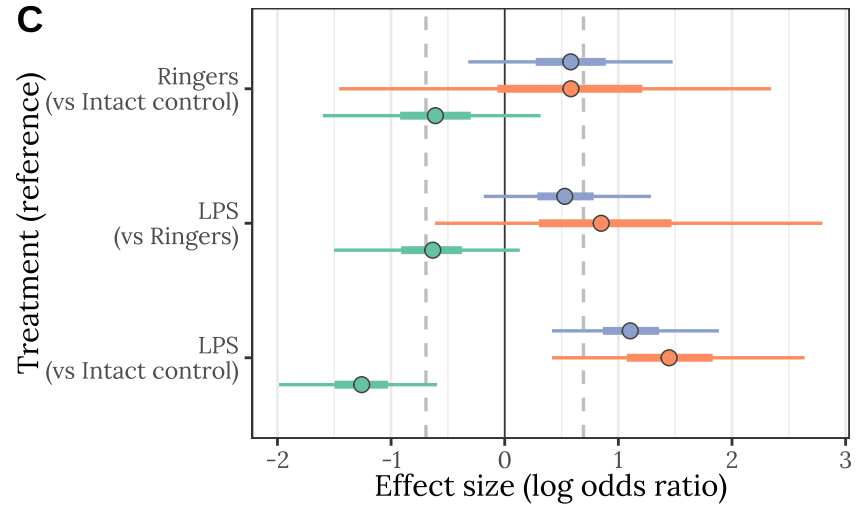

tribution. Panel $(\mathbf{C})$ gives the posterior estimates of the effect size of each treatment, relative to one of the other treatments (whose name appears in parentheses), expressed as a log odds ratio (LOR). Positive LOR indicates that the percentage of bees showing this particular outcome is higher in the treatment than the control; for example, more bees left voluntarily (orange) or were forced out (blue) in the LPS treatment than in the intact control. The vertical lines mark $\mathrm{LOR}=0$, indicating no effect, and $\mathrm{LOR}= \pm \log (2)$, i.e. the point at which the odds are twice as high in one treatment as the other 
evidence that the immune challenge from LPS caused bees to leave even more often than bees that were wounded but not otherwise immune-challenged. Bees were also somewhat more likely to leave the hive if treated with Ringers, relative to the intact control $(\mathrm{PP}=0.072)$. There were corresponding, opposite treatment effects on the numbers of bees that were forced out, or left voluntarily (Fig. 1, Table S2), though the differences were more apparent for the 'Forced out' outcome (because this outcome was more common, increasing statistical power; Fig. 1A). In particular, bees that received LPS were non-significantly more likely to be forced out than those that received the Ringers control $(\mathrm{PP}=0.074)$. The treatment effect size was quite large (Fig. 1B, Table S2); for example, the log odds ratio comparing the frequency of the 'Forced out' outcome between the Intact control and LPS treatments was 1.12, indicating that LPS-treated bees were forced out 3.1-fold more often (i.e. $e^{1.12}$ ).

\section{Experiment 2: Role of cuticular odours in behavioural responses to immune challenge}

In the preliminary GLMM analysis, there was a significant effect of treatment (Wald $\chi^{2}$ test; $p=0.0155$ ) on the proportion of bees leaving the hive. More bees were left in the group that was coated with odours from LPS-treated bees, relative to the control group coated with odours from Ringers-treated bees.

Figure 2A uses the results of the Bayesian MLM to display the posterior estimates of the percentages of bees from each treatment group that stayed inside the hive, left voluntarily, or were forced out, while Fig. 2B shows the standardised effect size of the LPS treatment, for each of the three response categories. Table S3 shows the model's parameter estimates, and Table $\mathrm{S} 4$ shows the results of planned contrasts comparing each pair of treatments.

Figures 2A-B and Tables S3-4 illustrate that bees coated with chemical extracts from LPS-treated bees were forced out of the hive significantly more often than were those treated with chemical extracts from Ringers-treated bees $(\mathrm{PP}=0.0046)$. The effect size of LPS was quite large (Log odds ratio: 1.08; Fig. 2B, Table S4), indicating that LPSperfumed bees were forced out 2.9 -fold more often (i.e. $\left.e^{1.08}\right)$ than the Ringers-perfumed controls. Interestingly, there was no significant difference in the rate at which the two treatment groups left the hive voluntarily $(\mathrm{PP}=0.29)$, as expected given that the focal bees in experiment 2 were intact and not immune-challenged.

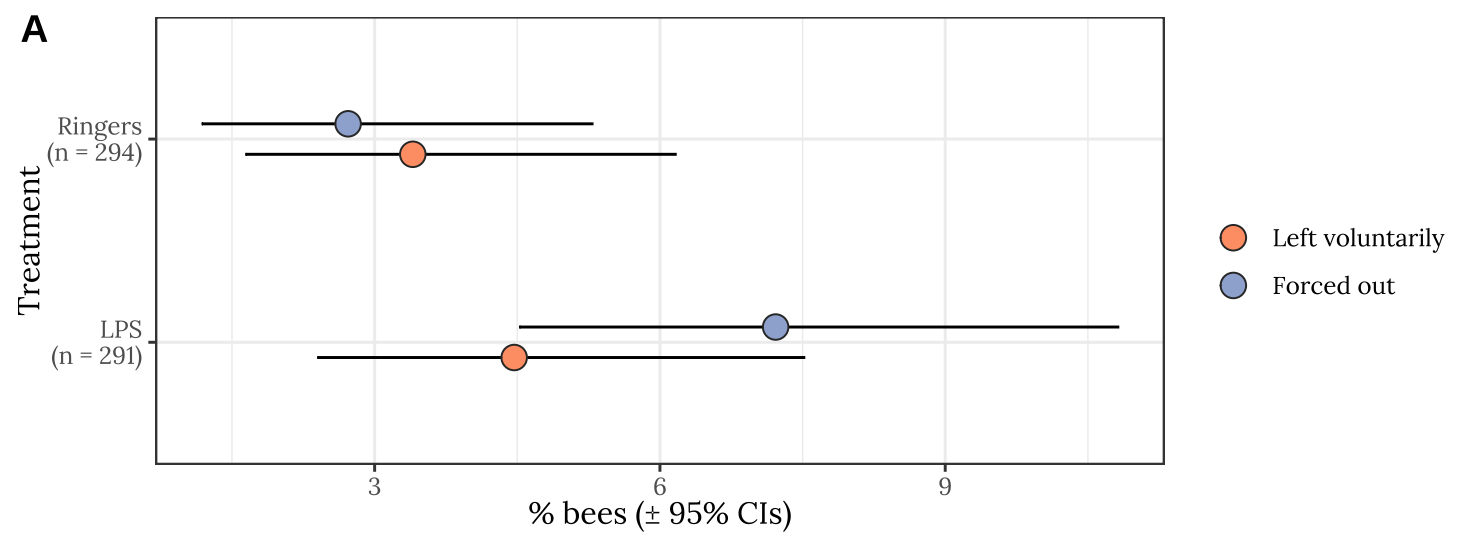

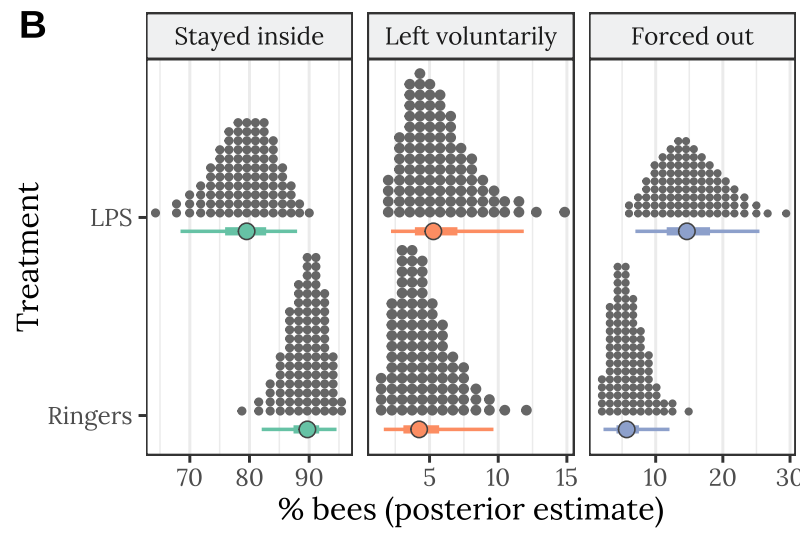

Fig. 2 Results of experiment 2 ( $n=585$ bees). Panels (A)-(B) display the same information as the corresponding panels in Fig. 1. Panel (C) gives the posterior estimates of the effect size (log odds ratio) of the

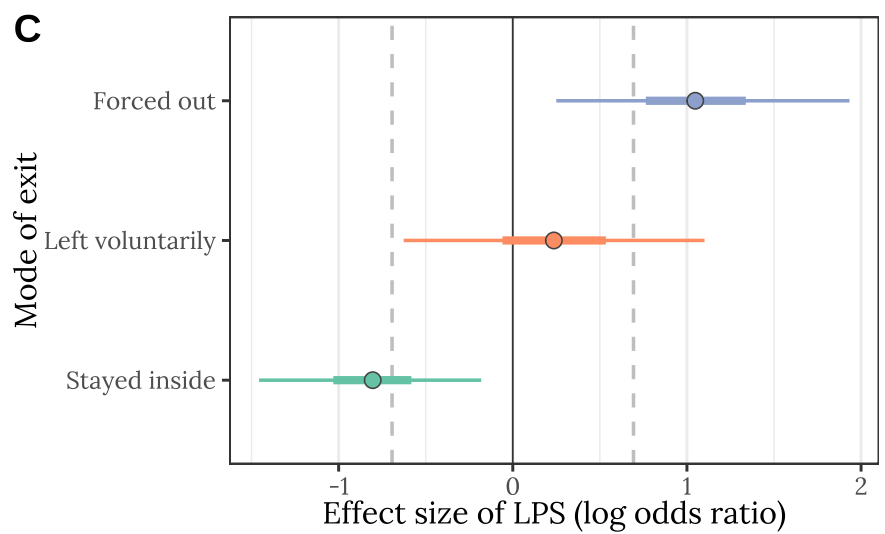

LPS treatment as a log odds ratio, for each of the three possible outcomes; the details are the same as for Fig. 1C 


\section{Experiment 3: 'Social distancing' following immune challenge}

Figure 3 illustrates that pairs of bees in which one individual had been treated with LPS spent less time in close contact than pairs in which one individual had received Ringers. Figure 3A shows histograms of the raw data, illustrating that bees in the control group more often spent $>90 \%$ of the 3.5-h observation in close contact, while LPS treatment bees were over-represented among pairs that spent a lot of time apart. Tables S5-6 give the associated statistical results; the mean percentage time in close contact (Fig. 3B) differed statistically significantly between treatments $(\mathrm{PP}=0.032)$, and the effect size was moderate (log odds ratio: 0.37).

\section{Discussion}

Experiment 1 revealed that bees treated with LPS were more likely to leave the hive compared to the intact control and (marginally non-significantly; $p=0.052$ ) the Ringers control. Most of the bees that left the hive in experiment 1 were forced out by other colony members, though many appeared to leave voluntarily, by walking out and then dropping to the ground without any apparent involvement from other workers. Very few left by flying. In this sense, our results differ from those of an earlier study (Rueppell et al 2010), which reported that bees with experimentally impaired health instead left the hive by flight. This discrepancy may reflect the studies' different methodologies; for example, Rueppell et al. challenged bees with $\mathrm{CO}_{2}$ or hydroxyurea, while we used an LPS-coated pin. We frequently found the hive-exiting bees dead on the same or subsequent days, and many were carried off by Vespid wasps

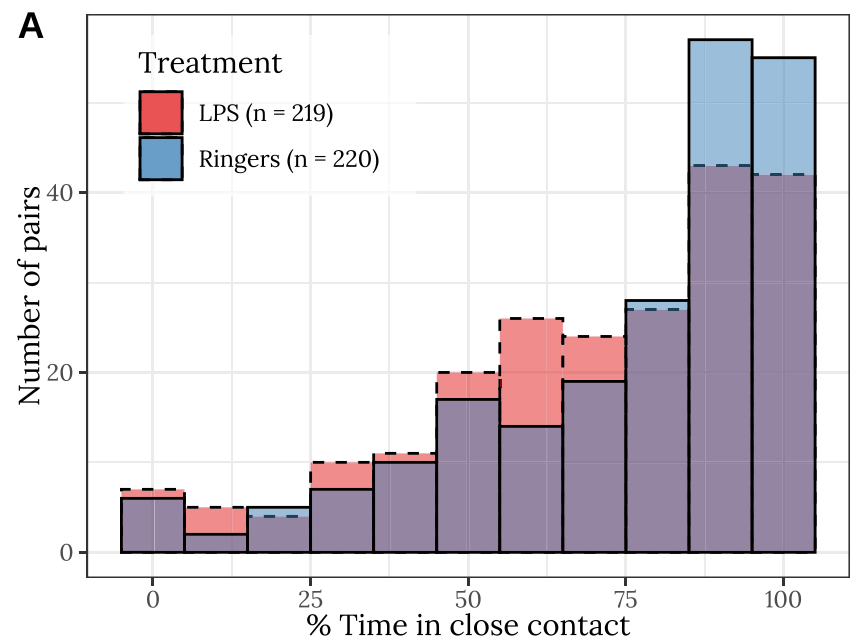

Fig. 3 Results of experiment 3 ( $n=439$ pairs of bees). Panel (A) shows the frequency distribution of the percentage time in close contact, for pairs of bees from the LPS treatment and the Ringers control. within seconds of dropping clear of the hive, illustrating that the hive-exiting behaviour is unlikely to represent an attempt to temporarily self-quarantine. From experiment 1 , we conclude that bees that were experimentally wounded and/or immunestimulated bees tend to leave the hive, both with and without any apparent involvement from other workers. Therefore, our study reaffirms the existence of both the 'altruistic self-removal' reported by Rueppell et al. (2010) and the forceful ejection of sick individuals observed by Richard et al. (2008) in honey bees. The relative importance of wounding (which occurred in the Ringers control and LPS treatment, but not the intact control) and immune challenge (LPS only) in generating these behavioural responses is unclear, given the borderline-significant difference between the Ringers and LPS treatments, though the ordering of the treatment means (Fig. 1A) implies that both wounding and immune challenge may affect behaviour.

The removal of immune-stimulated bees by their nestmates implies that sick individuals produce signals or cues which allow them to be identified for removal from the colony. We hypothesised that at least some of these signals/cues would be olfactory, since Hymenoptera rapidly develop a distinct chemical profile after an immune challenge (Richard et al 2008; Holman et al 2010). We tested this hypothesis in experiment 2 and found that bees coated with hexane-soluble chemicals extracted from the body surface of immune-challenged bees were ejected from the colony about threefold more often than controls, which were treated with chemicals from Ringerstreated bees. Interestingly, there was no treatment effect on the rate at which bees left the hive voluntarily. We hypothesise that this difference stems from the mismatch created in experiment 2 between the personal information held by the experimental individuals (which correctly perceived themselves to be healthy), and the chemical cues on their body surface (which

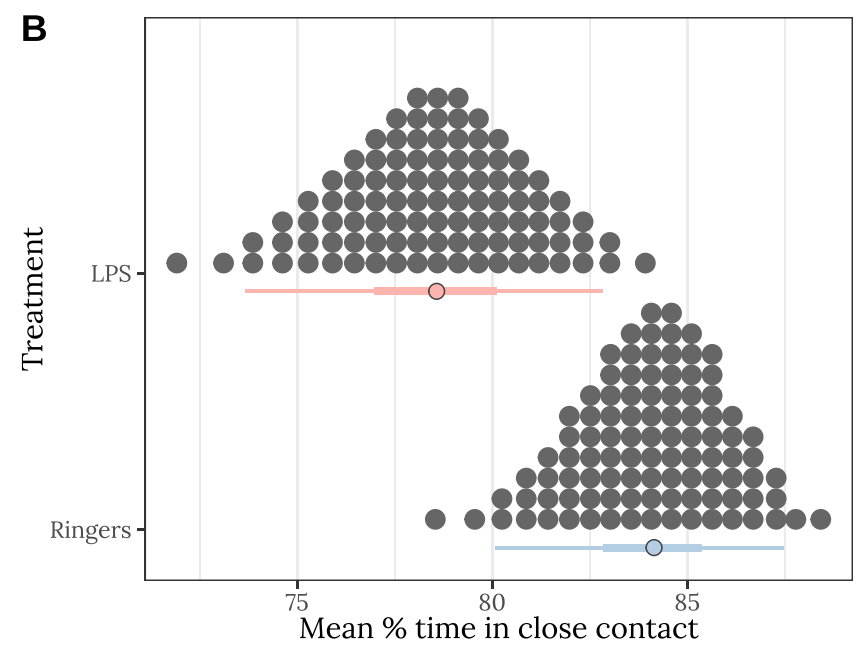

Panel (B) shows the posterior estimates of the mean percentage time spent in close contact; the details of the quantile dot plot and error bars are the same as described for Fig. 1 
came from immune-challenged individuals), causing the bees to be forced out by their nestmates, which perceived the experimental individuals to be sick.

Motivated by past findings that immune-challenged ants (Bos et al 2012) and bees (Richard et al 2008; Kazlauskas et al 2016) engage in fewer social interactions, experiment 3 tested whether pairs of bees in which one member had received an immune challenge spent less time in close contact than control pairs. We recorded a statistically significant decline in the proportion of time spent in contact, suggesting a behavioural response to immune challenge in the treated individual, the healthy individual paired with them, or both. A previous study recorded that bees directed more aggression and grooming behaviours towards immune-challenged bees (Richard et al 2008); behavioural effects like this could underpin our results. Another study recorded that LPS-treated bees showed reduced locomotion and antennated other individuals less often, which might also explain our results. Such 'sickness behaviour' occurs in non-social insects (e.g. Sullivan et al 2016), which suggests that it might confer fitness benefits unrelated to sociality (such as preserving energy), or might simply be a nonadaptive consequence of pathophysiology. However, in social species, sickness behaviour might also provide kin-selected benefits if it limits disease transmission to genealogical relatives (Kazlauskas et al 2016).

The chemical cues that distinguish healthy and immunechallenged individuals remain to be determined. Cuticular hydrocarbons ( $\mathrm{CHCs}$ ) are one likely possibility, given that honey bees utilise $\mathrm{CHCs}$ for chemical recognition in several other contexts (e.g. van Zweden and d'Ettorre 2010). Furthermore, the CHC profile changes rapidly following an immune challenge, in both eusocial and non-eusocial insects. For example, honey bee workers injected with Gram-negative bacteria began to produce relatively more unsaturated and shorter-chained hydrocarbons within $6 \mathrm{~h}$, and there were concomitant changes in the expression of genes involved in CHC biosynthesis (Richard et al 2012). Another reason to suspect CHCs is that the insect innate immune response involves changes in the expression of genes that have pleiotropic effects on lipid metabolism/ homeostasis, such as those in the IMD pathway (e.g. Pull et al 2018; Kamareddine et al 2018), such that there are a plausible mechanistic links between the $\mathrm{CHC}$ profile and immune status. However, no study has yet manipulated the CHC profile without confounds-our study and earlier, similar experiments (Richard et al 2008) involved solvent washes rather than treatment with $\mathrm{CHCs}$ specifically-so the involvement of CHCs remains to be demonstrated conclusively. Additionally, experiments have indicated that the gut microbiome of honey bees influences the composition of their CHC profile (Vernier et al 2020). It is plausible that immunostimulation with LPS (or a live pathogen) would change the gut microbiome (e.g. by inducing proliferation of granulocytes or antimicrobial peptide production; Rodrigues et al 2010) and hence the CHC profile.

Another outstanding question is whether the changes in the external chemical cues of immune-challenged bees represent an adaptation, or simply a non-adaptive consequence of other processes (i.e. a 'spandrel'; Gould and Lewontin 1979). Under the adaptive hypothesis, sick bees that purposefully signal their illness would be the superorganismal equivalent of infected vertebrate cells, which use MHC class I proteins to present antigens to cytotoxic $\mathrm{T}$ cells, which then destroy the infected cell. Presumably, the antigen-presenting system evolved adaptively (Forni et al 2014); it could be framed as a kin-selected adaptation because the self-sacrificing cells confer a benefit to genetically identical cells in the same body. Under the non-adaptive model, immune-challenged bees might produce modified chemical cues for reasons other than eliciting their own removal, e.g. because of pleiotropic links between immunity and metabolism (Kamareddine et al 2018); the key feature distinguishing these two hypotheses is the presence of a net inclusive fitness benefit to workers that solicit their own removal. In support of the non-adaptive hypothesis, immune challenge has also been found to affect the CHC profile of non-social insects that appear to have no need for social immunity (e.g. the beetle Tenebrio molitor; Nielsen and Holman 2012). To begin establishing whether chemical signalling of immune status has been favoured by kin selection, one could test whether social insects undergo uniquely strong chemical or behavioural changes following an immune challenge, relative to non-social insects, in a formal phylogenetic study.

Supplementary Information The online version contains supplementary material available at https://doi.org/10.1007/s00265-022-03139-z.

Acknowledgements We are grateful to Jean-Pierre Scheerlinck, Heidi Wong, and Daisy Kocher for assisting with the experiments.

Funding This work benefited from an Australian Research Council Discovery Project grant to LH (DP170100772).

Data and code availability Raw data and documented R code and output is available at lukeholman.github.io/social_immunity.

\section{Declarations}

Conflict of interest The authors declare no competing interests.

Open Access This article is licensed under a Creative Commons Attribution 4.0 International License, which permits use, sharing, adaptation, distribution and reproduction in any medium or format, as long as you give appropriate credit to the original author(s) and the source, provide a link to the Creative Commons licence, and indicate if changes were made. The images or other third party material in this article are included in the article's Creative Commons licence, unless indicated otherwise in a credit line to the material. If material is not included in the article's Creative Commons licence and your intended use is not 
permitted by statutory regulation or exceeds the permitted use, you will need to obtain permission directly from the copyright holder. To view a copy of this licence, visit http://creativecommons.org/licenses/by/4.0/.

\section{References}

Aubert A, Richard FJ (2008) Social management of LPS-induced inflammation in Formica polyctena ants. Brain Behav Immun 22(6):833-837

Baracchi D, Fadda A, Turillazzi S (2012) Evidence for antiseptic behaviour towards sick adult bees in honey bee colonies. J Insect Physiol 58(12):1589-1596

Bos N, Lefèvre T, Jensen A, d'Ettorre P (2012) Sick ants become unsociable. J Evol Biol 25(2):342-351

Bot AN, Currie CR, Hart AG, Boomsma JJ (2001) Waste management in leaf-cutting ants. Ethol Ecol Evol 13(3):225-237

Bürkner PC (2017) Advanced Bayesian multilevel modeling with the R package brms. arXiv preprint: 170511123

Christe P, Oppliger A, Bancalà F, Castella G, Chapuisat M (2003) Evidence for collective medication in ants. Ecol Lett 6(1):19-22

Cremer S, Armitage SA, Schmid-Hempel P (2007) Social immunity. Curr Biol 17(16):R693-R702

Forni D, Cagliani R, Tresoldi C, Pozzoli U, De Gioia L, Filippi G, Riva S, Menozzi G, Colleoni M, Biasin M, et al (2014) An evolutionary analysis of antigen processing and presentation across different timescales reveals pervasive selection. PLoS Genetics 10(3):e1004,189

Gould SJ, Lewontin RC (1979) The spandrels of San Marco and the Panglossian paradigm: a critique of the adaptationist programme. Proc R Soc Lond B 205(1161):581-598

Greco MK, Hoffmann D, Dollin A, Duncan M, Spooner-Hart R, Neumann P (2010) The alternative pharaoh approach: stingless bees mummify beetle parasites alive. Naturwissenschaften 97(3):319-323

Greene MJ, Gordon DM (2003) Cuticular hydrocarbons inform task decisions. Nature 423(6935):32-32

Hamilton WD (1964) The genetical evolution of social behaviour. II Journal of Theoretical Biology 7(1):17-52

Hart AG, Ratnieks FL (2001) Task partitioning, division of labour and nest compartmentalisation collectively isolate hazardous waste in the leafcutting ant Atta cephalotes. Behav Ecol Sociobiol 49(5):387-392

Heinze J, Walter B (2010) Moribund ants leave their nests to die in social isolation. Curr Biol 20(3):249-252

Holman L (2018) Queen pheromones and reproductive division of labor: a meta-analysis. Behav Ecol 29(6):1199-1209

Holman L, Jørgensen CG, Nielsen J, d'Ettorre P (2010) Identification of an ant queen pheromone regulating worker sterility. Proceedings of the Royal Society b: Biological Sciences 277(1701):3793-3800

Hughes DP, Brodeur J, Thomas F (2012) Host manipulation by parasites. Oxford University Press

Imler JL, Tauszig S, Jouanguy E, Forestier C, Hoffmann JA (2000) LPS-induced immune response in Drosophila. J Endotoxin Res 6(6):459-462

Kamareddine L, Robins WP, Berkey CD, Mekalanos JJ, Watnick PI (2018) The Drosophila immune deficiency pathway modulates enteroendocrine function and host metabolism. Cell Metab 28(3):449-462

Kazlauskas N, Klappenbach M, Depino AM, Locatelli FF (2016) Sickness behavior in honey bees. Front Physiol 7:261

López-Uribe MM, Sconiers WB, Frank SD, Dunn RR, Tarpy DR (2016) Reduced cellular immune response in social insect lineages. Biology Letters 12(3):20150,984
McElreath R (2020) Statistical rethinking: A Bayesian course with examples in $\mathrm{R}$ and Stan. CRC Press, London

Naug D, Camazine S (2002) The role of colony organization on pathogen transmission in social insects. J Theor Biol 215(4):427-439

Nielsen ML, Holman L (2012) Terminal investment in multiple sexual signals: immune-challenged males produce more attractive pheromones. Funct Ecol 26(1):20-28

Page P, Lin Z, Buawangpong N, Zheng H, Hu F, Neumann P, Chantawannakul P, Dietemann V (2016) Social apoptosis in honey bee superorganisms. Sci Rep 6(1):1-6

Pie MR, Rosengaus RB, Traniello JF (2004) Nest architecture, activity pattern, worker density and the dynamics of disease transmission in social insects. J Theor Biol 226(1):45-51

Pull CD, Ugelvig LV, Wiesenhofer F, Grasse AV, Tragust S, Schmitt T, Brown MJ, Cremer S (2018) Destructive disinfection of infected brood prevents systemic disease spread in ant colonies. eLife 7:e32,073

Richard FJ, Hunt J (2013) Intracolony chemical communication in social insects. Insectes Soc 60(3):275-291

Richard FJ, Aubert A, Grozinger CM (2008) Modulation of social interactions by immune stimulation in honey bee, Apis mellifera, workers. BMC Biol 6(1):50

Richard FJ, Holt HL, Grozinger CM (2012) Effects of immunostimulation on social behavior, chemical communication and genomewide gene expression in honey bee workers (Apis mellifera). BMC Genomics 13(1):558

Rodrigues J, Brayner FA, Alves LC, Dixit R, Barillas-Mury C (2010) Hemocyte differentiation mediates innate immune memory in Anopheles gambiae mosquitoes. Science 329(5997):1353-1355

Rueppell O, Hayworth MK, Ross N (2010) Altruistic self-removal of health-compromised honey bee workers from their hive. J Evol Biol 23(7):1538-1546

Simone-Finstrom M, Spivak M (2010) Propolis and bee health: the natural history and significance of resin use by honeybees. Apidologie 41(3):295-311

Smith AA, Hölldobler B, Liebig J (2012) Queen-specific signals and worker punishment in the ant Aphaenogaster cockerelli: the role of the Dufour's gland. Anim Behav 83(3):587-593

Spivak M (1996) Honey bee hygienic behavior and defense against Varroa jacobsoni. Apidologie 27(4):245-260

Stow A, Briscoe D, Gillings M, Holley M, Smith S, Leys R, Silberbauer T, Turnbull C, Beattie A (2007) Antimicrobial defences increase with sociality in bees. Biol Let 3:422-424

Sullivan K, Fairn E, Adamo SA (2016) Sickness behaviour in the cricket Gryllus texensis: comparison with animals across phyla. Behav Proc 128:134-143

Turnbull C, Hoggard S, Gillings M, Palmer C, Stow A, Beattie D, Briscoe D, Smith S, Wilson P, Beattie A (2011) Antimicrobial strength increases with group size: implications for social evolution. Biol Let 7:249-252

van Zweden JS, d'Ettorre P (2010) Nestmate recognition in social insects and the role of hydrocarbons. In: Blomquist GJ, Bagnères AG (eds) Insect Hydrocarbons: Biology, Biochemistry and Chemical Ecology, Cambridge University Press, Cambridge

Vernier CL, Chin IM, Adu-Oppong B, Krupp JJ, Levine J, Dantas G, Ben-Shahar Y (2020) The gut microbiome defines social group membership in honey bee colonies. Science Advances 6(42):eabd3431

Publisher's Note Springer Nature remains neutral with regard to jurisdictional claims in published maps and institutional affiliations. 